\title{
Accident technique et panne sociale : le cas des aérogénérateurs d'Électricité de France des années 1950-1960
}

Technical accidents and social breakdowns: The case of EDF wind turbines in the 1950 's and 1960's

\section{Philippe Bruyerre}

\section{OpenEdition Journals}

Édition électronique

URL : http://journals.openedition.org/artefact/4671

DOI : 10.4000/artefact.4671

ISSN : 2606-9245

\section{Éditeur :}

Association Artefact. Techniques histoire et sciences humaines, Presses universitaires du Midi

\section{Édition imprimée}

Date de publication : 15 décembre 2019

Pagination : 143-162

ISBN : 978-2-8107-0667-9

ISSN : 2273-0753

\section{Référence électronique}

Philippe Bruyerre, «Accident technique et panne sociale : le cas des aérogénérateurs d'Électricité de France des années 1950-1960 », Artefact [En ligne], 11 | 2019, mis en ligne le 27 novembre 2020, consulté le 29 novembre 2020. URL : http://journals.openedition.org/artefact/4671 ; DOI : https:// doi.org/10.4000/artefact.4671

\section{cc) $(1) \odot$}

Artefact, Techniques, histoire et sciences humaines est mise à disposition selon les termes de la Licence Creative Commons Attribution - Pas d'Utilisation Commerciale - Pas de Modification 4.0 International. 


\section{Accident technique et panne sociale : le cas des aérogénérateurs d'Électricité de France des années 1950-1960}

\section{Philippe Bruyerre}

\section{Résumé}

Accidents et pannes sont de première importance pour les « démonstrateurs ", ces technologies testées en vraie grandeur avant leur mise sur le marché, notamment dans le domaine de l'énergie. Les incidents techniques peuvent s'accumuler entraînant coûts et délais ; sans soutien social, ces démonstrateurs risquent de disparaître. Faut-il alors parler d'accident technique résultant de l'accumulation d'incidents techniques ou de mise en panne sociale liée à la perte du soutien social qui a permis leur mise en œuvre initiale ? Cette question est posée à partir d'une étude de cas, celle des aérogénérateurs d’EDF entre 1958 et 1963.

\section{Mots-clés}

démonstrateur, énergie, histoire, éolienne, recherche et développement

95 Philippe Bruyerre, «Accident technique et panne sociale : le cas des aérogénérateurs d'Électricité de France des années 1950-1960 », Artefact, 11, 2019, p. 143-162. 


\section{Technical accidents and social breakdowns: The case of EDF wind turbines in the 1950's and 1960's}

\section{Abstract}

Accidents and breakdowns are crucial events for "demonstrators"-technologies which are tested in full scale before they are commercialized, especially in the energy sector. When technical problems accumulate, they lead to added costs and delays; indeed, without social support, demonstrators can very well disappear. When that happens, should we speak of a technical accident, resulting from the accumulation of smaller technical incidents, or of a social failure, linked to the loss of social support that allowed the initial implementation of the technology? To answer this question, this article examines the case study of EDF wind turbines between 1958 and 1963. 


\section{Introduction}

Certaines techniques exigent, avant d'être commercialisées, d'être testées en vraie grandeur sur ce que l'on appelle des démonstrateurs. À ce stade, l'objet technique est proche de sa maturité et doit faire la preuve de son bon fonctionnement dans des conditions aussi proches que possible des futures conditions d'usage. C'est notamment le cas dans le secteur de l'énergie où les effets de taille jouent un rôle important : on parle ainsi de " tête de série » pour la centrale nucléaire EPR, de " démonstrateur pré-industriel » pour l'hydrolienne ${ }^{1}$. Cette zone grise de l'objet technique, non encore diffusable mais complètement défini dans sa fonction et ses structures, est considérée de façon imagée comme une traversée de "vallées de la mort ", technologique et économique ${ }^{2}$. Dans ce contexte de démonstrateur, les pannes et accidents sont à la fois attendus et redoutés, induisant délais et coûts de remise en état.

Quand les incidents sont trop nombreux, et les coûts trop élevés, la décision d'arrêter le démonstrateur tombe, justifiée le plus souvent sur la base de critères technico-économiques. Ces critères objectifs sont-ils cependant les seuls à prendre en compte pour comprendre cet abandon ? Il semble difficile de laisser de côté un autre type de justification, celle de la perte du soutien social dont a bénéficié initialement ce démonstrateur. Pour arriver à ce stade de démonstration, la technique en question a en effet obtenu de multiples soutiens institutionnels, politiques voire culturels : la technique était jugée faisable et prometteuse, répondant à un besoin humain défini, justifiant un investissement de plusieurs années d'études préalables.

Le démonstrateur, par son existence même, est un objet à la fois technique et social et la décision d'arrêt (ou de poursuite) est elle aussi technique et sociale. À côté d'une raison technique avec l'accident technique comme accumulation insoutenable d'incidents, ne faut-il pas aussi envisager une raison sociale, une mise en panne du démonstrateur, à la manière du navigateur qui oriente les voiles pour que son bateau n'avance plus ? Pour approcher cette problématique, cet article prend comme étude de cas celui des

1. Société française d'énergie nucléaire (SFEN), 2018, p. 25 - Hydrolienne (production d'énergie avec les courants marins) : Sabella SAS, https://www.sabella.bzh/fr/les-projets/d10 (consulté le 3 juillet 2019).

2. European Commission and London Economics, 2016. 
démonstrations d'aérogénérateurs qu'effectue EDF de 1958 à 1963. Après une présentation des aspects techniques puis du contexte social et économique de ces démonstrateurs, les modalités d'arrêt de ces démonstrateurs éoliens en 1964 et de leur disparition totale en 1966 seront examinées.

\section{Les démonstrations d'aérogénérateurs}

"Quel est cet étrange appareil ? » titre en première page le Parisien Libéré dans son édition du 5 février $1958^{3}$. La réponse est donnée : «la $1^{\text {re éolienne }}$ de France de l'E.D.F. ». Cette machine est installée sur l'une des deux "Stations d'Études de l'Énergie du Vent », toutes deux mises en œuvre par Électricité de France [EDF] de 1958 à 1963, l'une à Nogent-le-Roi (Eure et Loir), la seconde à Saint-Rémy-des-Landes (Cotentin).

Conçue par B.E.S.T. (Bureau d'études scientifiques et techniques), un bureau d'études dirigé par Lucien Romani qui rassemble des spécialistes de mécanique des fluides et d'aérodynamique, l'aérogénérateur de Nogentle-Roi a un diamètre de 30,2 m et une puissance de $640 \mathrm{~kW}$ (Fig. 1). Commandées en 1950 par EDF, les études prennent plusieurs années avec essais dans différentes souffleries (ONERA, Poitiers, Toulouse), test de résistance d'une pale, maquette en site réel à Saint-Cyr-l'École ${ }^{4} .$. Toutes les précautions sont prises, les calculs validés, les essais effectués sur la base des techniques éprouvées de l'aérodynamique et de l'électrotechnique. Certes le B.E.S.T. met en œuvre ses propres brevets, mais ils portent avant tout sur des arrangements structurels (mât pivotant et profilé...) visant à minimiser les coûts.

En parallèle, Neyrpic propose à EDF une machine de $20 \mathrm{~m}$ de diamètre et de $200 \mathrm{~kW}$ de puissance en septembre $1954^{5}$, le marché étant signé le 7 février 1955. Cette entreprise grenobloise, industriel reconnu de l'hydraulique, s'appuie dans le domaine des éoliennes sur l'expertise de l'ingénieur-conseil Louis $\operatorname{Vadot}^{6}$ et de l'ingénieur Édouard Gion. Les essais

3. Lamarre, 14 février 1958.

4. Rebuffet et Mirande, 1950 ; Rauline, 1956.

5. Argand, 22 Mars 1955.

6. Vadot, oct. 1958 et fév. 1959. 
préliminaires sont ici aussi nombreux : essai en soufflerie à Toulouse, prototype de $8 \mathrm{~m}$ de diamètre installé sur site réel à Saint-Nizier en $1955^{7}$.

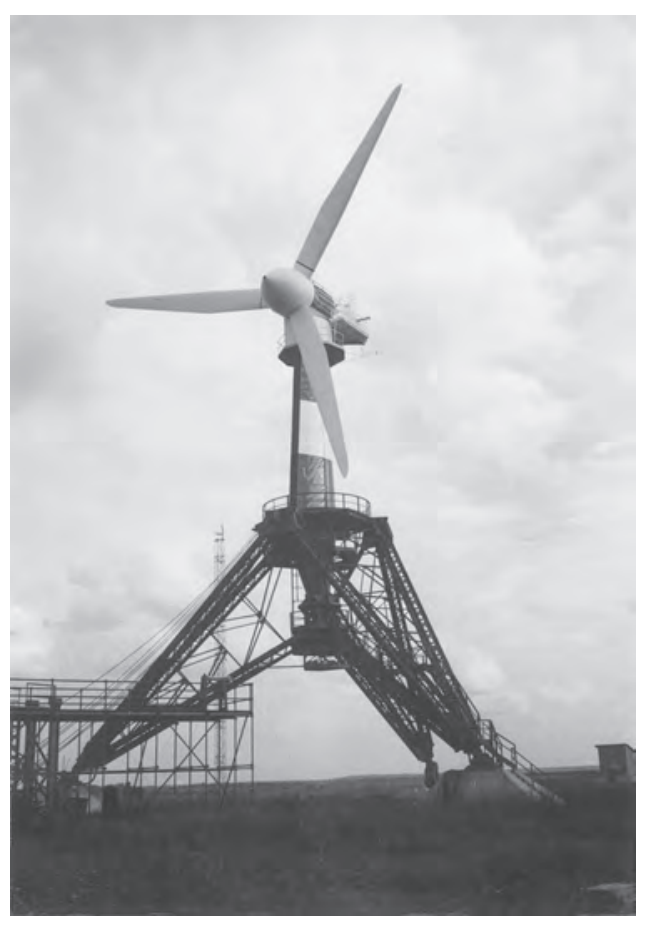

Fig. 1. - Aérogénérateur de Nogent-le-Roi

Source : Jean-Luc Cavey

Le 2 avril 1958, l'aérogénérateur B.E.S.T. débite sur le réseau et atteint une puissance de $200 \mathrm{~kW}$. L'aérogénérateur est ensuite en marche continue et de nombreuses défaillances sont constatées : "désordres " dans le circuit d'huile, "jeu " dans la fixation de l'orienteur, " dégâts " mécaniques sur l'arbre. Tout cela conduit à l'incident du 18 novembre 1959 entraînant la destruction de l'alternateur. À Saint-Rémy-des-Landes, l'aérogénérateur Neyrpic de 132 kW est installé à la fin de l'année 1957 et mis en service en avril 1958. Des essais de vibrations sont réalisés en décembre 1958 et la campagne d'essais démarre en janvier 1959. La machine connaît différents incidents notamment sur le système d'orientation de la machine face au vent. En avril, ce système est démonté et remplacé par une orientation sous le vent. Suite à des essais à pleine puissance, une pale casse le 28 juin 1959. Le second semestre de 1959 est une période difficile pour les deux aérogénérateurs. Les incidents survenus semblent être considérés comme des 
" erreurs de jeunesse " et les essais se poursuivent tout en développant en parallèle de nouvelles conceptions : hélice rapide $e^{8}$ et machine birotor ${ }^{9}$ par B.E.S.T., aérogénérateur de $1000 \mathrm{~kW}$ par Neyrpic ${ }^{10}$.

La machine B.E.S.T. est remise en état et couplée au réseau le 13 avril 1960. De nombreux incidents surviennent : fuites d'huile, échauffement du deuxième multiplicateur (septembre 1960), jeu dans l'accouplement (septembre 1961), craquements aux pieds de pale. Des mesures de vibration réalisées à la fin de l'année 1961 mettent en évidence une vibration de flexion torsion avec un phénomène de battement ${ }^{11}$. L'hélice est démontée le 12 avril 1962. Du 2 avril 1958 au 12 avril 1962, ce sont quatre années pendant lesquelles l'aérogénérateur a fonctionné 5428 heures dont seulement 1780 heures en production, soit 6,1\% du temps.

De son côté, l'aérogénérateur Neyrpic est équipé de pales en fibre de verre en juin $1960^{12}$. Celles-ci remplacent les anciennes pales en aluminium. De novembre 1962 à mars 1966, la machine produira $700000 \mathrm{kWh}$ alors que sa production était estimée à $500000 \mathrm{kWh}$ par an.

L'année 1962 est l'année charnière pour ces aérogénérateurs après plus de quatre ans de tests. Malgré des essais en soufflerie peu probants, les efforts sont poursuivis avec l'installation d'une hélice rapide à Nogent-le-Roi qui doit permettre de supprimer un des deux multiplicateurs et préfigurer l'aérogénérateur birotor en projet, testé en soufflerie en 1960. Installée en avril 1962, une pale casse le 5 septembre 1963 après 307 heures de fonctionnement et l'éolienne est définitivement arrêtée. Comme le dira Lucien Romani plus tard : "Cette hélice, trop poussée, s'est rompue par fatigue à la suite de vibrations auto-entretenues ${ }^{13}$."

À Saint-Rémy-des-Landes, la décision est prise d'installer une machine de plus grande taille, 35 mètres de diamètre et $1000 \mathrm{~kW}$ de puissance, qui arrive sur site en 1962 pour être raccordée au réseau le 13 juin 1963. Moins d'un an plus tard, l'éolienne est arrêtée le 10 juin 1964, après deux mille heures de fonctionnement, suite au « bruit insolite provoqué par la

\footnotetext{
8. B.E.S.T., 1957.

9. B.E.S.T., 1963 , p. 1.

10. EDF, Service des Etudes et Recherches, octobre 1960.

11. Vallée, 1961.

12. Vallée, 1960.

13. Romani, 1977, p. 31.
} 
partie tournante du labyrinthe du palier aval PV [Petite Vitesse ${ }^{14}$ ". Le lendemain, les techniciens constatent la détérioration des rouleaux du roulement et l'aérogénérateur est définitivement arrêté.

L'année 1964 est celle de la fin des deux démonstrateurs (trois en fait, car la machine Neyrpic est toujours présente). Rien n'est entrepris pendant plus de deux ans, et encore plus étonnant, il faut attendre le mois de juin 1966 pour qu'une visite technique soit organisée à la recherche des causes " ayant probablement conduit à l'accident et entraîné l'arrêt de l'aérogénérateur ${ }^{15}$. " À la fin de l'année 1966, les deux aérogénérateurs sont démontés et ferraillés : seuls subsistent de ces deux stations d'essais les quatre plots de fondations de l'aérogénérateur B.E.S.T.

Dans un article publié dans La Houille Blanche en 1975, la seule publication officielle, EDF tire différentes conclusions. Le comportement est jugé bon et « les seuls incidents rencontrés étaient d'origine mécanique, ne mettant pas en cause le principe d'utilisation de l'hélice ${ }^{16}$ ». Pour EDF, " les problèmes se situent au niveau des multiplicateurs de vitesse entre l'hélice et la génératrice électrique. Il y a donc un effort à fournir du côté électro-technique. " Comparant les deux stations d'étude, EDF considère que la machine B.E.S.T. " a posé, puis permis de résoudre de nombreux petits problèmes technologiques ; néanmoins elle a montré qu'il n'était pas impossible de faire fonctionner sans difficulté des machines de diamètres de l'ordre de 40 à $60 \mathrm{~m}$. ". Par rapport à la machine Neyrpic, " elle a plus joué le rôle de prototype technologique que la machine de $1000 \mathrm{~kW}$ implantée près du littoral de la Manche ».

\section{Le développement de l'énergie éolienne à EDF de 1943 à 1963}

Ces démonstrateurs sont l'aboutissement d'un programme de développement de l'énergie éolienne commencé dès le début des années 1940 dans le cadre du Comité d'organisation de l'énergie électrique sur la base d'une note de 1943 de l'ingénieur Pierre Ailleret ${ }^{17}$, X-Ponts et Supélec : mise en

14. Vallée, 1964.

15. EDF (Service d'Etude Générales Nucléaires), 1966.

16. Bonnefille, 1975.

17. Ailleret, 1943. 
place par le gouvernement en 1943 d'un Comité technique de l'énergie des vents ${ }^{18}$, création en novembre 1944 par des industriels privés de l'électricité d'une société dédiée, la SETAM ${ }^{19}$, et publications d'articles techniques ${ }^{20}$. À la création d'EDF en 1946, Pierre Ailleret, devenu directeur des études et recherches, crée une division du vent dirigée par le centralien André Argand.

La mise en place d'une Direction des études et recherches (DER) vise à donner à l'entreprise les moyens de concevoir son appareil de production et de transport, à contrôler le prix et la qualité des constructeurs de matériel qui eux ne sont pas nationalisés et enfin, sur le modèle américain de General Electric ${ }^{21}$, à développer de la vraie recherche et non pas de la "fausse " recherche (recherche appliquée) comme le font les entreprises françaises selon les termes d'un rapport d'experts du CNRS de $1942^{22}$. Les ingénieurs de la DER sont investis d'une mission de service public visant à contrôler les entreprises privées à la recherche du pur profit (les " trusts» vilipendés dans les années de guerre). Certains anciens d'EDF témoigneront plus tard d'un " anticapitalisme ${ }^{23}$ » des ingénieurs de l'entreprise publique qui attire des "polytechniciens-fonctionnaires ${ }^{24}$ ", X-Ponts spécialisés en hydraulique en particulier.

Dans un contexte de croissance très forte à la sortie de la Seconde Guerre mondiale, la " loi d'Ailleret " prévoit le doublement de la consommation tous les dix ans. Les moyens de production existants (hydraulique, thermique) ne suffiront pas et le risque de «disette énergétique » se profile à l'horizon : les ressources en charbon semblent atteindre leurs limites, l'hydraulique va être saturée ${ }^{25}$ et d'autres sources de production d'électricité doivent être développées.

18. Journal Officiel, 1943, p. 2940.

19. "Société d'études d'aéromoteurs", 17 novembre 1944. Archives EDF, Boîte 712 218. Sociétés actionnaires : Union d'électricité, Société auxiliaire d'études et d'entreprises, Compagnie française des conduites d'eau et Société du crédit foncier colonial et de banque.

20. Ailleret, 1944 ; Ailleret, 1946.

21. Smith, 1990.

22. Picard, 1987 , p. 10 et 19.

23. Picard, Beltran et Bungener, 1984. Dans un exemplaire annoté de sa main, Pierre Massé indique en remarque "plutôt leur impérialisme".

24. Picard, Beltran et Bungener, 1985, p. 27.

25. Ailleret, 1948, p. 173. 
La division du vent, chargée de développer des moyens de productions d'énergie éolienne de grande puissance (1 $000 \mathrm{~kW}$ minimum) raccordés au réseau, s'inscrit dans cette logique. Elle traduit la littérature technique étrangère dans le domaine (États-Unis avec la machine de Granpa’s Knob de $1000 \mathrm{~kW}$ en 1941, une référence pour EDF), visite les expériences étrangères, teste des machines existantes (aéromoteur danois Lykkegaard en 1948), participe à des réunions de spécialistes ${ }^{26}$, étudie les brevets et reçoit les inventeurs.

Le 21 janvier 1949, EDF présente au Comité technique de l'énergie des vents un rapport sur les aéromoteurs en cours d'étude ${ }^{27}$. Sur la base de l'expérience réalisée aux États-Unis et des mesures de vent effectuées en France, EDF envisage des machines de plus de $1000 \mathrm{~kW}$ et annonce avoir confié quatre études de détail à différents inventeurs : aéromoteur B.E.S.T. ${ }^{28}$, aéromoteurs Chevreau-Deville ${ }^{29}$, aéromoteur S.E.B.I. (Société d'études et de brevets industriels) de M. Andreau ${ }^{30}$ et barrage aéromoteur Morel ${ }^{31}$. Les ingénieurs de la division du vent valident les différents choix de conception, les essais réalisés et les prix : ils sont co-concepteurs. Dans le cas de B.E.S.T., le choix d'une génératrice électrique de type synchrone (alternateur) est ainsi le fait d'EDF, Lucien Romani lui préférant une génératrice asynchrone dans son étude de $1947^{32}$. Dans le cas de Neyrpic, le passage d'une orientation face au vent à une orientation sous le vent en 1958 est suggéré par $\mathrm{EDF}^{33}$.

Les moyens financiers dont dispose cette division du vent sont très importants : l'aérogénérateur B.E.S.T. coûtera quelque 216 millions de francs (essais préalables et appareillage de mesure inclus), soit 4,4 millions d'euros 2018 et celui de Neyrpic de 132 kW, 30 millions de francs $(560000$ euros 2018), selon une note d'André Argand de $1956^{34}$. Le coût total des trois aérogénérateurs (celui de B.E.S.T. et les deux de Neyrpic) correspond à près de $30 \%$ du montant total des investissements de la DER sur la

26. Organisation for European Economic Co-operation [OEEC], 1954.

27. Ailleret, 1949.

28. André et B.E.S.T., 1948 ; Romani et B.E.S.T., 1948 ; Romani 1949.

29. Société d'Etudes des Procédés Chevreau et Deville, 1941.

30. Andreau, 1943.

31. Morel, 1946.

32. Romani, 1947.

33. Témoignage de Georges Vallée, ingénieur de la division du vent.

34. Argand, 1956. 
période de 1947 à $1961^{35}$, une valeur très importante qui ne prend pas en compte les investissements dans les mesures de vent (350 mesures seront effectuées dans le monde entier).

Tous ces travaux se réalisent dans le plus grand secret au sein d'une communauté d'une dizaine d'ingénieurs (division du vent d'EDF-DER, B.E.S.T. et Neyrpic). Les publications sont rares et ne concernent que l'activité de mesure du vent et l'évaluation du potentiel éolien des sites. Les relations avec la presse sont inexistantes sauf pour annoncer le démarrage des essais. Les sites ne sont guère visités sauf par des sommités comme Francis Perrin, haut-commissaire à l'Énergie atomique qui juge la puissance de ces engins peu significative. Même dans la maison EDF, au plus haut niveau de l'étatmajor, ces travaux ne sont guère soutenus, comme l'exprime en 1955 le président du conseil d'administration d'EDF :

L'Électricité de France n'envisage pas encore d'utiliser industriellement d'autres modes d'énergie. L'énergie solaire ou celle du vent, pour ne parler que de celles-là, ne paraissent, en effet, susceptibles dans la métropole, que d'applications limitées, pour le moment ${ }^{36}$.

Le monde politique est lui aussi peu informé : dans leur avis sur le deuxième Plan de modernisation (1954-1957), les sénateurs ne citent l'énergie éolienne que " pour mémoire, les réalisations sur le sol métropolitain ou d'outre-mer étant actuellement au stade expérimental ${ }^{37}$ ".

À la fin de l'année 1963, la décision de dissoudre la division du vent est prise en interne par Alexis Dejou et son adjoint Michel Banal qui ont pris la succession de Pierre Ailleret à la tête de la DER. Le motif officiel invoqué est économique avec la baisse continue du pétrole et l'émergence du nucléaire ${ }^{38}$, des raisons à considérer avec prudence : de 1960 à 1966, le prix du pétrole passe de 14,71 à 12,73 dollars le baril, une baisse certes, mais relativement peu importante. Quant au nucléaire (0,9 MW installés en 1965), il est très loin d'être compétitif malgré les annonces tonitruantes

35. Sources : coût total des trois aérogénérateurs selon Bonnefille, 1975 ; montant des investissements de la DER selon Picard, 1987, annexe 1, p. 143.

36. Flouret, 1955, p. 7.

37. Coudé du Foresto, Cornat et Bonnet, 1956. Georges Bonnet, sénateur de la Lozère de 1955 à 1973, est chef de service à l'Électricité de France. Les deux autres sénateurs sont ingénieurs.

38. Banal, 1996, p. 158. 
de General Electric ${ }^{39}$. Les "seules contraintes économiques » ne suffisent pas à expliquer l'arrêt des éoliennes comme le dit Magnien, directeur de la DER en 1980 :

C $[\ldots]$ l'interruption du programme [d'aérogénérateurs] suivi par notre établissement [DER] jusqu'en 1964 est présentée à plusieurs reprises comme résultant des seules contraintes économiques de l'époque. Il s'agit bien là d'un élément important mais cette décision résultait aussi d'une évolution très lente et difficile de la fiabilité des équipements que nous avions constatée ${ }^{40}$.

Dans ce contexte d' ' évolution très lente " de la technique éolienne, le fait majeur est le départ au début des années 1960 de Pierre Ailleret qui tient à bout de bras l'énergie éolienne depuis 1943 et la prise de pouvoir à la DER d'une nouvelle génération d'ingénieurs comme Michel Banal, Alexis Dejou ou Michel Hug. L'éolien est privé de support institutionnel et financier et la division du vent disparait à la fin de l'année 1963 à peine six mois après la mise en service de la seconde machine Neyrpic et trois mois après la rupture de l'hélice rapide à Nogent-le-Roi.

\section{Accident technique et panne sociale}

Jean-Marcel Jeanneney, ministre de l'Industrie, expose la politique du gouvernement en matière d'énergie en 1959: "assurer l'approvisionnement du pays en énergie à un prix aussi bas que possible " sans que "notre balance des comptes ne soit trop lourdement grevée par des importations ${ }^{41}$ ". Sécurité d'approvisionnement, compétitivité économique et indépendance nationale sont d'ailleurs toujours les trois thèmes qui structurent la politique énergétique aujourd'hui, auxquels on peut ajouter le changement climatique. Le critère "à un prix aussi bas que possible " est déterminant pour l'énergie éolienne et tous les acteurs éoliens l'ont en tête. Il ne s'agit pas seulement de faire des essais d'une technologie, mais de préfigurer des aérogénérateurs produisant une énergie compétitive avec celle du charbon et de l'hydraulique. Les choix de conception sont d'ailleurs constamment guidés par la recherche de la compétitivité économique.

39. Burness, 1980.

40. Magnien, 1980.

41. Jeanneney, 1959, p. 1900. 
Dans une note de $1956^{42}$, André Argand, après avoir détaillé les prix de l'aérogénérateur B.E.S.T., évalue le "Prix en petites séries d'appareils analogues simplifiés " à 70 millions de francs pour une production en année moyenne de $1500000 \mathrm{kWh}$ dans le Pas-de-Calais, le Cotentin, le Finistère et $2000000 \mathrm{kWh}$ dans les Pyrénées Orientales. En marge, une note manuscrite indique : " 2 millions de $\mathrm{kWh}$ pour 70 millions de frs $35 \mathrm{f}^{43}$ et c'est du bon fil de l'eau ». En avril 1959, alors que les premiers tests sont encourageants, le bilan de la société B.E.S.T. fait apparaître comme actif incorporel le "Contrat de Royalties avec E.D.F. (aérogénérateur de grande dimension) " pour un montant de 30000000 francs, représentant les trois quarts du bilan de la société.

Les machines installées sont donc plus que des prototypes, ce sont des démonstrateurs, des "têtes de série " d'une future machine industrielle (First of a Kind ou FOAK) dans le cadre d'une activité de RD\&D, Recherche, Développement et Démonstration ${ }^{44}$. Dans cette industrie de l'énergie, le passage de l'idée à sa réalisation exige certes de la recherche et développement, mais aussi une phase de démonstration compte tenu des effets d'échelle entre un prototype de laboratoire et une machine de production. Ce qui marche à l'échelle du kilowatt ne marchera pas toujours à l'échelle du mégawatt, voire du millier de mégawatts.

La technique de l'aérogénérateur raccordé au réseau électrique est connue depuis la fin des années 1920 : les Russes ont construit une machine de 200 kW en Crimée en 1931, les Américains une éolienne de 1000 kW dans le Vermont en 1941 (la référence pour Pierre Ailleret), et le danois Johannes Juul une machine de $200 \mathrm{~kW}$ au Danemark en 1956. Utilisant des techniques éprouvées (aviation, électrotechnique, mécanique), le niveau de maturité technologique de ces aérogénérateurs est élevé, de niveau 7 défini comme la "démonstration du système prototype en environnement opérationnel ${ }^{45}$ " (le niveau maximal de 9 correspond à une technique commerciale).

\footnotetext{
42. Argand, 1956.

43. 6,3 centimes d'euro, valeur 2018.

44. Agence Internationale de l'Energie [AIE], 2018. L'acronyme RD\&D fait l'objet de différentes interprétations : sur la page Internet de l'AIE, on trouve Research, Development and Deployment, https://www.iea.org/statistics/rdd/. Les Américains parlent de First-of-a-Kind (FOAK) et de Nthof-a-Kind (NOAK).

45. European Commission, 2016.
} 
Pour un tel démonstrateur, les pannes et accidents ont un statut particulier. Dans les rapports internes, il n'est fait état que d'incidents comme dans le rapport de Georges Vallée à Saint-Rémy-des-Landes ${ }^{46}:$ « le 23 janvier 1959, incident sur la tringlerie de commande », " le 10 février 1959 incident sur le collecteur orientation "; " 10 mars 1959 incident collage de l'électro du déchargeur "... Même la rupture d'une pale est considérée comme un incident ${ }^{47}$. Ce n'est que dans un rapport de $1966^{48}$ que l'on parle d'accident comme évènement qui " a entraîné l'arrêt de l'aérogénérateur " alors qu'en 1962 il s'agissait d'un incident ${ }^{49}$. L'accumulation des incidents du démonstrateur devient accident (technique) de l'aérogénérateur après la décision (sociale) d'abandon.

Du point de vue technique, il n'existe que des incidents et des accidents comme dans l'échelle des événements nucléaires ${ }^{50}$, auxquels s'ajoutent des catastrophes dans le domaine des risques naturels et technologiques ${ }^{51}$. Dans ces échelles, les incidents se distinguent des accidents par les dommages qu'ils causent et les coûts afférents. À ces incidents et " accident " (technique), l'analyse qui vient d'être faite suggère d'associer la "panne " (sociale) en prenant ce mot dans son usage premier dans la navigation à voiles. Comme le dit Pierre Bouguer en 1757, un navire est en panne quand les marins se servent des voiles " pour rester, pour ainsi dire, dans la même place, lorsqu'ils veulent attendre quelque autre Navire, ou qu'ils ont quelque raison particulière de ne pas s'éloigner de là où ils sont ${ }^{52}$ ". La panne est ici une mise en arrêt volontaire de la machine pour quelque raison particulière, une décision humaine et sociale. C'est bien le cas des aérogénérateurs EDF, ces "navires sédentaires ${ }^{53}$ ", mis en panne en 1964, sabordés en 1966. Certains le regretteront deux décennies plus tard comme Michel Banal, adjoint de la DER à l'époque, l'exprime en 1987 :

Je suis moins fier d'avoir arrêté l'énergie du vent - que les marémotrices - dit celui-ci, une hélice du moulin de Nogent-le-Roi

\footnotetext{
46. Vallée, 1959a.

47. Vallée, 1959b.

48. Vallée, 1966.

49. Vallée, 1964.

50. International Atomic Energy Agency, 2014.

51. Ministère de l'Ecologie et du Développement Durable, 2003, p. 7.

52. Bouguer, 1757, p. 305-306.

53. Severac, 1978.
} 
s'est cassée, quant aux deux machines de Saint-Rémy-des-Landes que l'on s'apprêtait à exploiter, elles ont cassé elles aussi (1964). J'ai essayé de réparer la petite mais personne ne s'y est intéressé ${ }^{54}$.

Cette décision d'abandon est humaine, à la fois consciente et inconsciente si l'on suit l'analyse de Pierre Ailleret :

Nous ne sommes pas habitués dans la plupart des métiers d'ingénieurs (sauf peut-être pour le génie civil) à ce que les chiffres soient mieux justifiés par l'autorité d'un homme que par les calculs numériques et nous avons souvent tendance à réclamer la justification chiffrée par écrit. Mais quand un grand chirurgien annonce la nécessité d'une grosse opération, qui oserait lui demander un calcul justificatif mettant en évidence les diverses probabilités en cause?

Il est au fond de même dans la plupart des grandes décisions industrielles ${ }^{55}$.

L'ingénieur annonce l'accident et prend la décision industrielle d'arrêter les démonstrateurs comme le chirurgien annonce une grosse opération. Il s'appuie sur des faits, des rapports écrits, des calculs, mais aussi sur une intime conviction. Il sait aussi que ces éoliennes sont de trop faible puissance par rapport aux enjeux, que l'installation de 500 éoliennes de $2 \mathrm{MW}$ est bien plus complexe pour EDF qu'une seule centrale de $1000 \mathrm{MW}$, qu’à la " disette énergétique " d'après-guerre se substitue le " tournant commercial $^{56}$ ». Perdant les soutiens qui avaient justifié leur mise en œuvre, les aérogénérateurs sont en panne sociale, ou plutôt mis en panne intentionnellement, les empêchant de surmonter les incidents techniques.

\section{Conclusion}

Pour les démonstrateurs, les pannes et accidents sont des événements importants puisqu'ils déterminent la survie de la technique à démontrer. Dans le cas des aérogénérateurs analysé ci-dessus, la distinction faite entre accident technique et panne sociale permet de mieux comprendre ce que les ingénieurs de la division du vent font. Si les machines installées connaissent de nombreux incidents, la décision d'arrêt est avant tout une

54. Picard, 1987, p. 46.

55. Ailleret, 1954, p. 9-10.

56. Wieviorka et Trinh, 1989, p. 49. 
décision institutionnelle liée à l'évolution de la DER d'EDF après le départ de Pierre Ailleret, son chef historique. Il ne s'agit pas de relativiser les incidents techniques, mais ceux-ci sont surmontables : la première machine Neyrpic donne satisfaction, la casse d'un multiplicateur sur la seconde machine Neyrpic est loin d'être insurmontable, et l'aérogénérateur de Nogent-le-Roi est victime d'une sorte de fuite en avant avec le test risqué d'une hélice " rapide ", sans remettre en cause la technique elle-même.

La mise en panne des aérogénérateurs dès la fin de l'année 1963 avec la suppression de la division du vent, le démantèlement complet des deux sites (et des trois éoliennes) en 1967 sont aussi des indices d'une volonté interne à la DER d'oublier un épisode de plus de quinze ans. La disparition de la quasi-totalité des archives EDF vient renforcer cette analyse et la découverte récente d'archives d'un ancien ingénieur de la division du vent et du directeur de la société B.E.S.T. a permis d'apporter un éclairage nouveau.

Au Danemark, à la même époque que celle des aérogénérateurs d'EDF, l'éolienne de Gedser ${ }^{57}$, fonctionnant depuis 1959, est elle aussi arrêtée en 1966 suite au décès de son concepteur, Johannes Juul, et du peu d'intérêt que lui porte la compagnie d'électricité SEAS, aux nombreuses défaillances, notamment des fuites d'huile (d'où son surnom de "moulin à huile "...), aux frais d'entretien supérieurs aux recettes de production d'électricité... Là aussi, l'éolienne est mise en panne, mais ne sera pas démantelée comme les aérogénérateurs français. Elle sera remise en route en $1976^{58}$ quand les Américains et les Danois s'intéresseront à nouveau à l'énergie éolienne dans le nouveau contexte de "crise de l'énergie ". Par ailleurs, les nombreuses publications de Johannes Juul seront une source d'inspiration pour les petits fabricants d'éoliennes danoises au début des années $1970^{59}$.

Cet exemple danois montre qu'une technique en panne dans un contexte donné peut redevenir en usage dans un autre contexte. Les incidents, pour peu qu'ils soient rendus publics, sont surmontables ou tout au moins constituent un retour d'expérience évitant de nouveaux déboires. Ce pourrait être l'ambition d'une histoire des techniques (mises) en panne que de reprendre ces démonstrateurs n'ayant pas survécu dans leur contexte, d'en analyser les incidents et accidents ainsi que le contexte social de leur mise en panne.

57. Danske Elværkers Forening, 1963.

58. Lundsager, Fransen et Christensen Clayton, 1980.

59. Maegaard Preben et al., 2013. 


\section{Sources}

\section{Archives nationales}

Romani Lucien, Avant-projet d'éolienne de $200 \mathrm{~kW}$ du type hélice-moulinet, B.E.S.T., 30 novembre 1947. Sous-direction de la sécurité de la navigation maritime, 19910305/2.

\section{EDF Archives}

Ailleret Pierre, L'énergie du vent, Note au C.O.E.E., Tapuscrit, 25 septembre 1943. Archives EDF, Boîte 890574.

Ailleret Pierre, L'Énergie du Vent - Position Actuelle du Problème - Recherches à faire, Paris, Syndicat Professionnel des Producteurs et Distributeurs d'Énergie Électrique, 31 mai, $1^{\text {er }}$ et 2 juin 1944. Boîte 890575.

Ailleret Pierre, "Rapport présenté par Monsieur Ailleret au Comité Technique des Vents le 21/1/1949 sur les aéromoteurs en cours d'étude à l'E.D.F. », 1949. Boîte 890575 .

Ailleret Pierre, "Prévisions d'avenir ", Bulletin de l'Union des Exploitations Électriques en Belgique, n 5, novembre 1954, p. 9-10. Boîte 890576.

Argand André, Compte Rendu des activités de la division "énergie du vent " pour 1954, note du 22 mars 1955, Ref. HV.2550 -AA/GB, p. 8. Boîte 852761.

Argand André, Note du 16 mars 1956 adressée à M. Chapouthier, Réf. HV.3130 - AA/GB. Boîte 852761.

B.E.S.T., "Hélice Éolienne à Grande Vitesse - Ensemble », plan n E-66-R2001, 20 décembre 1957. Boîte 852541.

Flouret Marc, "L'Électricité de France - Service National », EDF, 1955, p. 7. Boîte 789968.

"Société d'Études d'Aéromoteurs ", Société Anonyme d'Études au capital de 850000 francs, 17 novembre 1944. Boîte 712218 .

\section{Institut national de la propriété industrielle (INPI)}

AndrÉ Maurice (inventeur), B.E.S.T. (demandeur), "Perfectionnements aux éoliennes ", INPI, brevet FR1006473, déposé le 20 janvier 1948.

ANDrEAU Jean-Édouard, "Perfectionnements apportés aux moulinets producteurs de force motrice, notamment aux éoliennes ", INPI, brevet FR984961 (A), demandé le 19 octobre 1943 et $1 \mathrm{r}^{\mathrm{e}}$ addition $\mathrm{n}^{\circ}$ 55.893, demandée le 14 février 1944.

Morel Jean-Baptiste, "Appareil sans fin pour capter et transmettre l'énergie d'un fluide en mouvement ", INPI, brevet $n^{\circ}$ 938,150 demandé le 12 décembre 1946.

Société D’Études des Procédés Chevreau et Deville, "Aéromoteur ", Bureau Fédéral de la Propriété Intellectuelle, INPI, Confédération Suisse, brevet 
$\mathrm{n}^{\circ} 272377$, demande déposée le 27 décembre 1941 (priorités : France, 25 février 1941, 21 juillet 1942, et Allemagne, 4 décembre 1941).

\section{Office national d'études et de recherches aérospatiales (ONERA)}

Rebuffet Pierre et Mirande J., 1950, Étude de deux éoliennes E.D.F. en attaque normale et oblique, ONERA, Rapport PROB 287 AY, 30 mai 1950.

\section{Laboratoire aérodynamique Eiffel et Martin Peter}

B.E.S.T., Aérogénérateur B.E.S.T.-Romani $2 \times 1000 \mathrm{~kW}$-Avant-projet, Notice générale, rapport n R.259, 27 décembre 1963.

Magnien Maurice, directeur de la DER, Courrier à M. Henry Durand, Président du Commissariat à l'Énergie Solaire [COMES], 11 mars 1980.

Romani Lucien (inventeur), B.E.S.T. (demandeur), «Procédé de limitation du couple moteur des roues éoliennes et roue éolienne en comportant application ", II, brevet FR1006698(A), déposé le 11 février 1948.

Romani Lucien, «Perfectionnements aux éoliennes », brevet FR1008324, déposé le 14 octobre 1949.

Romani Lucien, Le vent, source d'énergie, Conférence présentée à l'Université de Montpellier en 1975, 1976 et 1977, tapuscrit, p. 31.

Rauline Georges, Aérogénérateur expérimental Ø 30 m-Étude Aérodynamique sur modèle réduit au 1/20, Paris, EDF, janvier 1956.

Rauline Georges, Aérogénérateur expérimental Type B.E.S.T. - Romani, Paris, EDF, mai 1958.

\section{Archives personnelles de Georges Vallée, ingénieur à la Division du Vent}

EDF - Service des Études et Recherches - Division du Vent, Aérogénérateur de 35 mètres de diamètre - Rapport d'études - Pièce $n^{\circ} 13$ - Note sur le réglage, les sécurités et le comptage de l'installation, Réf : EGi/FT - 11.903, octobre 1960.

EDF (Service d'Étude Générales Nucléaires), Compte rendu de visite à la Station d'Études de l'Énergie du Vent de St. Rémy des Landes (Manche), Réf : GVa/TB, juin 1966.

VAlléE Georges (1959b), Note sur les essais de fatigue exécutés du 29 juillet au 27 août 1959, Aérogénérateur NEYRPIC de $21.2 \mathrm{~m}$. de diamètre installé à St. Rémy des Landes, Manche, 18 août 1959.

VAlléE Georges (1959a), Aérogénérateur Neyrpic de 21,2 m de diamètre, Essais, $2 e$ Campagne, Station d'Études de l'Énergie du Vent de Saint Rémy des Landes (Manche), septembre 1959.

VAlléE Georges, Essais de réception provisoire du jeu de pales $n^{\circ} 2$ - Essais statiques mécaniques sur les trois pales réalisées en matière plastique comprenant du roving, des tissus de verre, du klegecel, des résines polyesters et constituant la nouvelle hélice éolienne, Station d'Études de l'Énergie du Vent de Saint Rémy des Landes (Manche), Aérogénérateur de 21,2 m de diamètre, juillet 1960. 
Vallée Georges, Aérogénérateur BEST-Romani de 800 kVA et 30,2 m de diamètre, Essais de vibrations sur la structure pivotante et le multiplicateur deuxième train, Station d'Études de l'Énergie du Vent de Nogent-le-Roi (Eure et Loir), décembre 1961.

VAllée Georges, Aérogénérateur Neyrpic de diamètre 35 m-Rapport préliminaire d'incident sur le palier aval petite vitesse du multiplicateur, EDF/DER, Division Énergie du Vent, juin 1964.

ValléE Georges, Compte Rendu de visite à la Station d'Études de l'Énergie du Vent de St. Rémy des Landes (Manche) - Objet : Examiner, au cours du démontage de l'aérogénérateur de $35 \mathrm{~m}$, le roulement aval (côté hélice) de l'arbre petite vitesse, Électricité de France, Service d'Études Générales Nucléaires, 8 et 9 juin 1966.

\section{Archives personnelles Édouard Gion, ingénieur chez Neyrpic}

Gion Edouard, Éolienne $\varnothing 8$ m de diamètre - Note sur les essais effectués à St-Nizier, Ets Neyrpic Grenoble, Rapport R. 4428, 5 octobre 1956.

VADOT Louis, Aérogénérateur 100-130 kW- Essais en souflerie et caractéristiques principales, rapport R. 4048, Laboratoire dauphinois d'Hydraulique et Neyrpic, 22 juin 1955.

\section{Documentation en ligne}

Lundsager P., Fransen S. et Christensen Clayton M., Analyis of Data from the Gedser Wind Turbine 1977-1979, RISO-M-2242, Risoe National Laboratory, Roskilde (Danemark), 1980 : https://orbit.dtu.dk/ws/files/33441311/ ris_m_2242.pdf.

SOCIÉTÉ FRANÇAISE D'ÉNERGIE NUCLÉAIRE (SFEN), Les coûts de production du nouveau nucléaire français, Contributions de la SFEN à la Programmation pluriannuelle de l'énergie, 2018 : http://www.sfen.org/sites/default/files/public/ atoms/files/les_couts_de_production_du_nouveau_nucleaire_francais.pdf.

\section{Bibliographie}

"Arrêtés du 19 octobre 1943 instituant un comité technique de l'énergie des mers et un comité technique de l'énergie des vents ", Journal Officiel de la République Française, $\mathrm{n}^{\circ} 274,75^{\mathrm{e}}$ année, lundi 15 et mardi 16 novembre 1943, p. 2940.

Agence Internationale de l'Énergie, IEA Energy Technology RD $\sigma D$ Budgets : overview, Paris, International Energy Agency, 2018.

Ailleret Pierre, "Énergie éolienne : sa valeur et la prospection des sites. ", Revue Générale de l'Électricité, vol. 55, mars 1946, p. 103-108.

Ailleret Pierre, "L'évolution probable de la production et de la distribution de l'énergie électrique ", Bulletin de la Société Française d'Électricité, 6e série, tome VIII, nº 80,1948 , p. 173-183. 
Association Française de Normalisation, Aéromoteurs - Aérogénérateurs de petite puissance, E 50-001, décembre 1956.

Banal Michel, "L'équipement électrique de la France à la Libération », in MorseL Henri (dir.), Histoire générale de l'électricité en France, tome III, Fayard, Paris, 1996, p. 97-182.

Bonnefille René, "Les réalisations d'Électricité de France concernant l'énergie éolienne ", La Houille Blanche, n 1, 1975, p. 44-65.

Bouguer Pierre, De la manouvre des vaisseaux, ou traité de méchanique et de dynamique, H.L. Guérin \& L.F. Delatour, Paris, 1757.

Burness Stuart H., Montgomery David W. and Quirk James P., «The Turnkey Era in Nuclear Power ", Land Economics, vol. 56, n 2, 1980, p. 188-202.

Coudé du Foresto Yvon, Cornat Henri et Bonnet Georges, Avis présenté au nom de la commission de la production industrielle sur le projet de loi, adopté par l'Assemblée nationale, portant approbation du deuxième pian de modernisation et d'équipement, Annexe n 316, tome I, session ordinaire de 1955-1956, séance du 6 mars 1956.

Cunty Guy, Éoliennes et aérogénérateurs : guide de l'énergie éolienne, Edisud, Aixen-Provence, 1982.

Danske Elværkers Forening (Association des producteurs d'électricité danois), Development, testing and operation of a $200 \mathrm{~kW}$ wind power station in Denmark, Report of the Wind Power Committee, Copenhague, 1963.

European Commission and London Economics, Innovative Financial Instruments for First-of-a-Kind, commercial-scale demonstration projects in the field of Energy, Directorate-General for Research \& Innovation, Brussels, 2016. European Commission, "Technology readiness levels (TRL) », in Horizon 2020 - Work programme 2016-2017, 20. General Annexes, Annexe G, Bruxelles, 2016.

INSEE, Classification des produits française, révision 2.1, août 2018.

International Atomic Energy Agency, The Use of the International Nuclear and Radiological Event Scale (INES) for Event Communication - Guidelines and Good Practices for Setting up a National Framework on the Effective Use of INES for Event Communication, IAEA, Vienne (Autriche), 2014.

Jeanneney Jean-Marcel, "Déclaration du Gouvernement sur sa politique en matière d'énergie ", Journal Officiel de la République Française, samedi 19 décembre 1959, p. 1900.

LAMARRE Louis, "Sur un plateau dominant la vallée de l'Eure, une éolienne géante pourra fabriquer de l'électricité pour toute une région ", Le Parisien Libéré, $15^{\mathrm{e}}$ année, $\mathrm{n}^{\circ} 4177,14$ février 1958. 
Maegaard Preben, Krenz Anna et Palz Wolfgang (dir.), Wind Power for the World - The Rise of Modern Wind Energy, Taylor \& Francis Group, LLC (coll. "Pan Stanford Series on Renewable Energy »), Boca Raton (Floride), 2013.

Ministère de L'Écologie eT Du Développement Durable, Les événements naturels dommageables en France et dans le monde en 2002 - Retour d'expérience, MEDD, Paris, 2003.

Organisation for European Economic Co-operation [OEEC], Technical Papers presented to the Wind Power Working Party, Majesty's Stationery Office, Londres, 1954.

Picard Jean-François, Beltran Alain et Bungener Martine, Mémoires d'une entreprise publique. Témoignages pour une histoire d'Électricité de France 19461981, CNRS, EDF, Paris, 1984.

Picard Jean-François, Beltran Alain et Bungener Martine, Histoires de l'EDF: comment se sont prises les décisions de 1946 à nos jours?, Paris, Dunod, 1985.

PICARD Jean-François, Recherche et industrie : témoignages sur quarante ans d'études et de recherches à Electricité de France, Eyrolles, Paris, 1987.

SEverac A., "L'Odyssée du moulin à vent. 8 siècles de navigation sédentaire sur toutes les mers du travail ", La Météorologie, vi ${ }^{\mathrm{e}}$ série, n ${ }^{\circ} 12$, mars 1978, p. 193-197.

Smith, John Kenly Jr., "The Scientific Tradition in American Industrial Research ", Technology and Culture, vol. 31, n 1, 1990, p. 121-131.

VADOT Louis, "La production d'énergie électrique par éoliennes ", La Houille Blanche, $\mathrm{n}^{\circ} 5$, octobre 1958 , p. 503-525 et $\mathrm{n}^{\circ} 1$, février 1959 , p. 3-14.

Wieviorka Michel et Trinh Sylvaine, Le modèle EDF. Essai de sociologie des organisations, La Découverte, Paris, 1989.

\section{L'auteur}

Ingénieur des Arts et Métiers, créateur d'entreprises, Philippe Bruyerre a travaillé de 1980 à 2007 dans le montage de projets éoliens. En 2017, il a soutenu une thèse en histoire des techniques à l'EHESS au Centre Alexandre-Koyré sous la direction de Liliane Hilaire-Pérez, proposant une histoire de long terme, technique et sociale, de l'« éolienne ». Sa thèse est en cours de publication aux Presses universitaires du Midi. Il a publié dernièrement : « Naissance des éoliennes : question de définition(s) », Technologie et innovation, vol. 4, 2019 (http://www.openscience.fr/ Naissance-des-eoliennes-question-de-definition-s) et « Moulin expérimental et électriciens ruraux : une transition énergétique locale au Danemark au début du $\mathrm{xx}^{\mathrm{e}}$ siècle », dans Pierre LAMARD et Nicolas Stoskopf (dir.), La transition énergétique : un concept historique?, Villeneuve d'Ascq, Presses universitaires du Septentrion, 2018, p. 143-162. Contact : pbruyerre@yahoo.fr 